\title{
Singularities of optimal attitude motions
}

\author{
James D. Biggs * \\ * Advanced Space Concepts Laboratory, Department of Mechanical \\ Engineering, University of Strathclyde, Glasgow. (e-mail: \\ james.biggs@strath.ac.uk).
}

\begin{abstract}
This paper considers the problem of planning optimal attitude motions for spacecraft. The extremal solutions that result from this optimization problem are characterized and their singularities identified. Following this these singularities are solved analytically inferring the form of particular optimal velocities. These particular solutions are then integrated and their corresponding motions derived independently of a local coordinate chart. These motions have the potential to be used as smooth, optimal reference trajectories for performing certain re-orientations for spacecraft.
\end{abstract}

Keywords: Motion Planning, attitude control, Maximum principle, Singularities.

\section{INTRODUCTION}

Methodologies for planning and controlling attitude maneuvers of spacecraft based on geometric techniques has a rich history and includes the application of quaternion algebra, Lie group theory and geometric control theory, see Wie (1998); Spindler (1996); Jurdjevic (1997); Leonard et. al. (1995) for a few examples. In this paper we consider the optimal attitude control problem posed in Spindler (1996) from the perspective of using these methods to plan optimal, smooth and practical motions for spacecraft.

Spindler (1996) defines a fixed end point optimal control problem for the attitude control of a rigid body with the angular velocities as the control inputs, where the cost function to be minimized is a quadratic function of the angular velocity components. In particular minimizing such a cost function is desirable during a spacecraft maneuver to keep angular velocity low because high spin rates can cause undesirable tumbling motions. In addition high spin rates make it hard to receive good tracking data to monitor the spacecraft's motion. Spindler (1996) then applies the Maximum Principle (see Jurdjevic (1997); Sussmann (1997)) to this optimal control problem to yield the appropriate Hamiltonian. Following this the corresponding Hamiltonian vector fields are derived to yield the necessary conditions for optimality.

In Spindler (1996) a special case of this optimal control problem is considered where the weights of the cost function are all equal and is representative of the mean square angular velocity. In this case the extremal curves are constant and their projections onto $S O(3)$ can be solved in closed-form. Following this trivial case Spindler (1996) gives a numerical example for the general case (the weights are not equal) and the corresponding extremal curves are solved numerically. In this paper we provide a detailed treatment of singularities that appear in the general solution of the extremal curves and proceed to solve them analytically. This investigation yields an additional set of analytically defined reference trajectories which alongside the constant extremal reference trajectories in Spindler (1996) could potentially be used in practical attitude interpolation problems for spacecraft.
Following the derivation of the extremals at these singularities, techniques based on Lie group theory are used to project them down to $S O(3)$ (see for example Jurdjevic (1997); Biggs (2010)). This procedure enables the derivation of globally defined solutions at the singularities of the extremal functions. These singular motions provide analytic expressions for rotational interpolation in a convenient simplistic form. Furthermore, these rotational motions could be used as practical reference trajectories for a spacecraft to track in order to perform particular manoeuvres.

\section{ATTITUDE MOTION PLANNING PROBLEM}

The orientation of a spacecraft is represented here by curves in the Special Orthogonal Group $S O(3)$ where $R(t) \in S O(3)$ and where the kinematics are described by the differential equation:

$$
\frac{d R(t)}{d t}=R(t)\left(\Omega_{1} A_{1}+\Omega_{2} A_{2}+\Omega_{3} A_{3}\right)
$$

where $\Omega_{1}, \Omega_{2}, \Omega_{3}$ are the angular velocities and where $A_{1}, A_{2}, A_{3}$ form a basis for the Lie algebra of $S O(3)$ :

$$
\begin{gathered}
A_{1}=\left(\begin{array}{ccc}
0 & 0 & 0 \\
0 & 0 & -1 \\
0 & 1 & 0
\end{array}\right), A_{2}=\left(\begin{array}{ccc}
0 & 0 & 1 \\
0 & 0 & 0 \\
-1 & 0 & 0
\end{array}\right) \\
A_{3}=\left(\begin{array}{ccc}
0 & -1 & 0 \\
1 & 0 & 0 \\
0 & 0 & 0
\end{array}\right)
\end{gathered}
$$

with the Lie bracket defined by $[X, Y]=X Y-Y X$ with $X, Y \in$ $\mathfrak{s o}(3)$. Therefore, we have $\left[A_{1}, A_{2}\right]=A_{3},\left[A_{2}, A_{3}\right]=A_{1}$ and $\left[A_{1}, A_{3}\right]=-A_{2} . A_{1}, A_{2}, A_{3}$ describe the infinitesimal motion of the spacecraft in the yaw, pitch, roll directions respectively.

The problem addressed by Spindler (1996) is formalized in the following problem statement:

\section{Motion Planning Problem}

Compute the optimal velocities and the corresponding rigid body motions $R(t) \in S O(3)$ defined by the kinematic equations (1) that minimizes the cost function:

$$
J=\frac{1}{2} \int_{0}^{T} c_{1} \Omega_{1}^{2}+c_{2} \Omega_{2}^{2}+c_{3} \Omega_{3}^{2} d t
$$


with the given boundary conditions $R(0)=R_{0}$ and $R(T)=R_{T}$, where $c_{1}, c_{2}, c_{3}$ are constant weights and $T$ is the final time.

In an optimal control problem where we desire to motion between two fixed orientations, the weights $c_{1}, c_{2}, c_{3}$ can be manipulated to achieve the final desired configuration. This problem can be viewed as an optimal control problem where the angular velocities are the control inputs. The tool used to tackle this Motion planning problem is the coordinate free Maximum Principle of optimal control as described in Spindler (1996); Jurdjevic (1997); Sussmann (1997). The Maximum Principle of optimal control identifies the appropriate leftinvariant Hamiltonian $H$ on the dual of the Lie algebra $\mathfrak{s o}(3)^{*}$. The Hamiltonian corresponding to (1) and (3) is written as (see for example Jurdjevic (1997)):

$$
H(p, u)=\sum_{i=1}^{3} \Omega_{i} p\left(R(t) A_{i}\right)-p_{0}\left(\frac{1}{2}\left(\sum_{i=1}^{3} c_{i} \Omega_{i}^{2}\right)\right)
$$

where $p(\cdot): T_{R(t)} S O(3) \mapsto \mathbb{R}$ such that $p\left(R(t) B_{i}\right), p\left(R(t) A_{i}\right)$ are scalar components of an element in $T_{R(t)}^{*} S O(3)$, where $p_{0} \geqslant 0$ is a fixed positive constant. The curves which satisfy the Hamiltonian (4) with $p_{0}=1$ are called regular extremals and with $p_{0}=0$ abnormal extremals. However, it has been shown in Spindler (1996) that for this optimal control problem no abnormal extremals exist. Following from the Maximum Principle and the fact that (4) is a concave function in $\Omega_{i}$ the optimal velocity inputs are given by $\frac{d H}{d \Omega_{i}}=0$ it follows that:

$$
\Omega_{i}=\frac{1}{c_{i}} p\left(R(t) A_{i}\right)
$$

As the configuration of the spacecraft is the Lie group $S O(3)$, the cotangent bundle $T^{*} S O(3)$ can be realized as the direct product $S O(3) \times \mathfrak{s o}(3)^{*}$ where $\mathfrak{s o}(3)^{*}$ is the dual of the Lie algebra Jurdjevic (1997). Therefore, the original Hamiltonian defined on $T^{*} S O(3)$ can be expressed as a reduced Hamiltonian on the dual of the Lie algebra $\mathfrak{s o}(3)^{*}$. We define the linear functions $M_{i}=p\left(R(t) A_{i}\right)=\hat{p}\left(A_{i}\right)$ for $i=1,2,3$, see Jurdjevic (1997). Therefore, from (16) it follows that the maximizing inputs are:

$$
\Omega_{i}=\frac{1}{c_{i}} M_{i}
$$

Substituting (6) into (4) gives the optimal Hamiltonian

$$
H=\frac{1}{2}\left(\frac{M_{1}^{2}}{c_{1}}+\frac{M_{2}^{2}}{c_{2}}+\frac{M_{3}^{2}}{c_{3}}\right)
$$

The necessary conditions for optimality are then computed by making use of the Poisson bracket defined in terms of the Lie bracket $\{\hat{p}(\cdot), \hat{p}(\cdot)\}=-\hat{p}([\cdot, \cdot])$ which yields:

$$
\begin{aligned}
& \dot{M}_{1}=\frac{c_{2}-c_{3}}{c_{2} c_{3}} M_{2} M_{3} \\
& \dot{M}_{2}=\frac{c_{3}-c_{1}}{c_{1} c_{3}} M_{1} M_{3} \\
& \dot{M}_{3}=\frac{c_{1}-c_{2}}{c_{1} c_{2}} M_{1} M_{2}
\end{aligned}
$$

where $M_{1}, M_{2}, M_{3} \in \mathfrak{s o}^{*}(3)$ are the extremal curves. It is interesting to note that if the weights (that would be set according to the final desired configuration) are set to correspond to the moments of inertia then the equations are exactly the Euler equations for a free-rigid body (see Whittaker (1999)). However, we note that these are a generalisation of these equations as the weights of the cost function are arbitrary. In order to characterize the extremal curves described by the equations (17) we conveniently express them in the form:

$$
\dot{M}_{i}=\frac{c_{j}-c_{k}}{c_{j} c_{k}} M_{j} M_{k}
$$

where $i=1,2,3, j=2,3,1$ and $k=3,1,2$ respectively. In the next section we observe that by exploiting an additional constant of motion inherent in all left-invariant Hamiltonian systems on $S O(3)$ the evolution of the extremal curves can be reduced to the analysis of a 1 dimensional ordinary differential equation and solved in terms of Jacobi elliptic functions. In Spindler (1996) only a numerical example of the general case is given.

\section{REDUCTION AND SOLUTION OF THE EXTREMAL CURVES}

The initial stage of the procedure is to derive analytic expressions for the optimal angular velocity history and the corresponding rotation matrix. Note that when $i=1$ then $j=2, k=3$ when $i=2$ then $j=3, k=1$ and when $i=3$, then $j=1, k=2$ : Theorem 1. The optimal angular velocities $\Omega_{i}^{*}$ that minimize the cost function (3) can be expressed in the form:

$$
\Omega_{i}^{*}=\frac{\sqrt{s_{i}}}{c_{i}} s n\left(\sqrt{\alpha s_{j}}+C_{i}, \frac{s_{i}}{s_{j}}\right)
$$

where $\operatorname{sn}(\cdot, \cdot)$ is a Jacobi elliptic function and where the constants $C_{i}$ are defined by

$$
C_{i}=s n^{-1}\left(\frac{c_{i} \Omega_{i}(0)}{\sqrt{s_{i}}}, \frac{s_{i}}{s_{j}}\right)
$$

with

$$
s_{i}=\frac{-\beta+\sqrt{\beta^{2}-4 \alpha \chi}}{2 \alpha} \quad s_{j}=\frac{-\beta-\sqrt{\beta^{2}-4 \alpha \chi}}{2 \alpha}
$$

and

$$
\begin{aligned}
\alpha & =-\frac{\left(c_{i}-c_{j}\right)\left(c_{i}-c_{k}\right)}{c_{i}^{2} c_{j} c_{k}} \\
\beta & =\frac{4 c_{j} c_{k} H-2 c_{i}\left(c_{j}+c_{k}\right) H+2 c_{i} K^{2}-\left(c_{j}+c_{k}\right) K^{2}}{c_{i} c_{j} c_{k}} \\
\chi & =-\frac{\left(2 c_{j} H-K^{2}\right)\left(2 c_{k} H-K^{2}\right)}{c_{j} c_{k}}
\end{aligned}
$$

with the conserved quantities $H$ and $K$ defined in terms of the initial angular velocities:

$$
\begin{aligned}
& H=\frac{1}{2}\left(c_{1} \Omega_{1}^{2}(0)+c_{2} \Omega_{2}^{2}(0)+c_{3} \Omega_{3}^{2}(0)\right) \\
& K^{2}=c_{1}^{2} \Omega_{1}^{2}(0)+c_{2}^{2} \Omega_{2}^{2}(0)+c_{3}^{2} \Omega_{3}^{2}(0)
\end{aligned}
$$

Proof.

the optimal Hamiltonian is given by:

$$
H=\frac{1}{2}\left(\frac{M_{1}^{2}}{c_{1}}+\frac{M_{2}^{2}}{c_{2}}+\frac{M_{3}^{2}}{c_{3}}\right)
$$

where the extremal curves $M_{1}, M_{2}, M_{3} \in \mathfrak{s o}^{*}(3)$ are defined in terms of the weights $c_{i}$ of the cost function and the optimal angular velocities:

$$
M_{i}=c_{i} \Omega_{i}^{*}
$$

the corresponding Hamiltonian vector fields are then given by the Poisson bracket (see Jurdjevic (1997) for details):

$$
\dot{M}_{i}=\left(\frac{c_{j}-c_{k}}{c_{j} c_{k}}\right) M_{j} M_{k}
$$

it is easily shown that the Casimir function:

$$
K^{2}=M_{1}^{2}+M_{2}^{2}+M_{3}^{2}
$$


is constant along the Hamiltonian flow. Illustrating the solution for $M_{1}$ the solutions for $M_{2}$ and $M_{3}$ follow analogously. From (17) we have:

$$
\left(\dot{M}_{1}\right)^{2}=\left(\frac{c_{2}-c_{3}}{c_{2} c_{3}}\right)^{2} M_{2}^{2} M_{3}^{2}
$$

then using the Hamiltonian (15) and the Casimir function (18) write $M_{2}$ and $M_{3}$ explicitly in terms of $M_{1}$ to yield:

$$
\begin{aligned}
& M_{2}^{2}=\frac{c_{2}\left(2 c_{3} H-K^{2}+M_{1}^{2}-\left(c_{3} M_{1}^{2}\right) / c_{1}\right)}{c_{3}-c_{2}} \\
& M_{3}^{2}=\frac{c_{3}\left(2 c_{2} H-K^{2}+M_{1}^{2}-\left(c_{2} M_{1}^{2}\right) / c_{1}\right)}{c_{2}-c_{3}}
\end{aligned}
$$

then substituting (20) into (19) and simplifying yields:

$$
\left(\dot{M}_{1}\right)^{2}=\alpha M_{1}^{4}+\beta M_{1}^{2}+\chi
$$

where the constants $\alpha, \beta$ and $\chi$ are defined by equation (13) with $i=1, j=2, k=3$. Defining the constants:

$$
s_{1}=\frac{-\beta+\sqrt{\beta^{2}-4 \alpha \chi}}{2 \alpha} \quad s_{2}=\frac{-\beta-\sqrt{\beta^{2}-4 \alpha \chi}}{2 \alpha}
$$

then equation (21) can be expressed as:

$$
\dot{M}_{1}=\left(s_{1}-M_{1}^{2}\right)\left(s_{2}-M_{1}^{2}\right)
$$

and therefore

$$
\int_{0}^{t} d t=\frac{1}{\sqrt{\alpha}} \int_{M_{1}(0)}^{M_{1}(t)} \frac{d M_{1}}{\sqrt{\left(s_{1}-M_{1}^{2}\right)\left(s_{2}-M_{1}^{2}\right)}}
$$

then with a change of variable $M_{1}=\sqrt{s_{1}} s n(u, m)$ and setting $m=\frac{s_{1}}{s_{2}}$ the equation (24) becomes:

$$
t=\frac{1}{\sqrt{\alpha s_{2}}} \int_{s n^{-1}\left(M_{1}(0) / \sqrt{s_{1}}, \frac{s_{1}}{s_{2}}\right)}^{s n^{-1}\left(M_{1}(t) / \sqrt{s_{1}}, \frac{s_{1}}{s_{2}}\right)} d u
$$

and therefore:

$$
M_{1}=\sqrt{s_{1}} \operatorname{sn}\left(\sqrt{\alpha s_{2}} t+C_{1}, \frac{s_{1}}{s_{2}}\right)
$$

where $C_{1}$ is defined in (11) and therefore from (16) the angular velocity is (10) $\square$.

Note that this analytic solution has been verified against numerical integration.

\section{SINGULARITIES OF THE EXTREMAL CURVES}

The differential equation (21) can be expressed as an Elliptic integral of the first kind:

$$
t=\int_{M_{i}(0)}^{M_{i}(T)} \frac{1}{\sqrt{\alpha M_{i}^{4}+\beta M_{i}^{2}+\chi}} d M_{i}
$$

In this paper we investigate the case at the singularities of this elliptic integral, that is where

$$
\alpha M_{i}^{4}+\beta M_{i}^{2}+\chi=0
$$

from (28) it can be seen that the singularities of the elliptic integral correspond to $\dot{M}_{i}=0$ in equation (21) and this fact is used to derive analytic expressions for this particular case. In this section we investigate the singularities of the extremal curves, that is where the elliptic integral (27) is not well defined. These singularities correspond to fixed points of the differential equation (21) and we define a root of this by the constant $M_{i}=s_{1}$. At this singularity we can solve the extremal curves $M_{j}$ and $M_{k}$ using the Casimir function such that:

$$
K^{2}-s_{1}^{2}=M_{j}^{2}+M_{k}^{2}
$$

as the left hand side of (29) is constant this suggests using polar coordinates for $M_{j}$ and $M_{k}$ :

$$
M_{j}=r \sin \theta, \quad M_{k}=r \cos \theta
$$

$r$ is given by substituting (48) into (29):

and $\theta$ is given as follows:

$$
r=\left(K^{2}-s_{1}^{2}\right)^{1 / 2}
$$

$$
\begin{gathered}
\theta=\arctan \left(\frac{M_{j}}{M_{k}}\right) \\
\dot{\theta}=\frac{M_{k} \dot{M}_{j}-M_{j} \dot{M}_{k}}{M_{j}^{2}+M_{k}^{2}}
\end{gathered}
$$

substituting in the values for $\dot{M}_{j}$ and $\dot{M}_{k}$ from (17) and simplifying gives:

$$
\dot{\theta}=s_{1}\left(\frac{K^{2}-c_{1} H}{c_{1}\left(K^{2}-s_{1}^{2}\right)}\right)
$$

therefore $\dot{\theta}$ is constant and it is convenient to write $\dot{\theta}=$ $C$. Assuming a particular solution such that the constant of integration is zero we have $\theta=C t$. Therefore, we can express the singular extremal curves explicitly as:

$$
M_{i}=s_{1} \quad M_{j}=r \sin C t, \quad M_{k}=r \cos C t
$$

where $s_{1}, r, C$ are constants. Therefore, at the singularities the extremal $M_{i}$ is constant and $M_{j}$ and $M_{k}$ are sinusoids. In the following section we illustrate a method for projecting the extremal curves onto $S O(3)$.

\section{INTEGRATION PROCEDURE}

To obtain the most convenient form for the rotation matrix the differential equations are expressed in Lax Pair form:

$$
\begin{aligned}
& \dot{L}(t)=[L(t), \nabla H] \\
& \frac{d R(t)}{d t}=R(t) \nabla H
\end{aligned}
$$

where $R(t) \in S O(3)$ with

$$
\begin{aligned}
& L(t)=M_{1} A_{1}+M_{2} A_{2}+M_{3} A_{3} \\
& \nabla H=\frac{M_{1}}{c_{1}} A_{1}+\frac{M_{2}}{c_{2}} A_{2}+\frac{M_{3}}{c_{3}} A_{3}
\end{aligned}
$$

this allows us to state the following theorem:

Theorem 2. The projection of the extremal curves $M_{1}, M_{2}, M_{3}$ (that satisfy the condition $M_{2}^{2}+M_{3}^{2}>0$ for all $t$ ) onto $S O(3)$ are of the form:

$$
R(t)=\left(\begin{array}{ccc}
b & a c & a d \\
a e & d f-b c e & -b d e-f c \\
-a f & d e+b c f & b d f-e c
\end{array}\right)
$$

where:

$$
\begin{gathered}
a=\frac{\sqrt{M_{2}^{2}+M_{3}^{2}}}{K}, \quad b=\frac{M_{1}}{K}, \\
c=\frac{M_{2}}{\sqrt{M_{2}^{2}+M_{3}^{2}}}, \quad d=\frac{M_{3}}{\sqrt{M_{2}^{2}+M_{3}^{2}}} \\
e=\sin \phi_{1} \quad f=\cos \phi_{1}
\end{gathered}
$$

where

$$
\frac{d \phi_{1}}{d t}=K \frac{H-\left(M_{1}^{2} / c_{1}\right)}{K^{2}-M_{1}^{2}}
$$

Proof.

For details, similar proofs can be found in Jurdjevic (1997); 
Biggs (2010) where it is shown that for a particular $R(0) \in$ $S O(3)$ that:

$$
L(t)=K R(t)^{-1} A_{1} R(t)
$$

Noting that the stabilizer of $K A_{1}$ under the adjoint action of $S O(3)$ is the one parameter group $G=\exp \left(\phi_{1} A_{1}\right)$, which describes rotations about the yaw axis, it is convenient to write:

$$
R(t)=\exp \left(\phi_{1} A_{1}\right) \exp \left(\phi_{2} A_{2}\right) \exp \left(\phi_{3} A_{1}\right)
$$

with the appropriate ranges of the angles defined by $\phi_{1}, \phi_{3} \in$ $(-\pi, \pi]$ and $\phi_{2} \in[0, \pi]$. Then substituting (42) into (41) and equating the left and right hand side of equation (41) it is shown that:

$$
L(t)=K\left(\begin{array}{ccc}
0 & -\cos \phi_{3} \sin \phi_{2} & \sin \phi_{2} \sin \phi_{3} \\
\cos \phi_{3} \sin \phi_{2} & 0 & -\cos \phi_{2} \\
-\sin \phi_{2} \sin \phi_{3} & \cos \phi_{2} & 0
\end{array}\right)
$$

which gives

furthermore

$$
M_{1}=K \cos \phi_{2}
$$

$$
\begin{aligned}
& M_{2}=K \sin \phi_{2} \sin \phi_{3} \\
& M_{3}=K \sin \phi_{2} \cos \phi_{3}
\end{aligned}
$$

dividing $M_{2}$ by $M_{3}$ in (45) gives $\phi_{3}$ in terms of the extremal solutions:

$$
\frac{M_{2}}{M_{3}}=\tan \phi_{3}
$$

then for the Euler angle $\phi_{3} \in(-\pi, \pi]$ it follows that

$$
\sin \phi_{3}=\frac{M_{2}}{\sqrt{M_{2}^{2}+M_{3}^{2}}}, \cos \phi_{3}=\frac{M_{3}}{\sqrt{M_{2}^{2}+M_{3}^{2}}}
$$

to obtain the expression for $\phi_{1}$ substitute (42) into $R(t)^{-1} \frac{d R(t)}{d t}=$ $\nabla H$ and equate the right and left hand side of this equation to yield (38).

The rotation matrix (38) is expressed in terms of the extremal curves and the Euler parameter $\phi_{1}$. In addition the derivative of $\phi_{1}$ is expressed in terms of the extremal curves. However, as $\phi_{1}$ itself is not expressed analytically the problem is not in a form suitable for parametric optimization. However, we proceed to use these expressions to derive an analytic expression for the rotation matrix corresponding to the optimal angular velocities at the singularity. A singular solution of the extremal curves has been shown to be:

$$
M_{1}=s_{1} \quad M_{2}=r \sin C t, \quad M_{3}=r \cos C t
$$

substituting (48) into (40) yields:

$$
\frac{d \phi_{1}}{d t}=K \frac{H-\left(s_{1}^{2} / c_{1}\right)}{K^{2}-s_{1}^{2}}
$$

as the right hand side is constant this yields:

$$
\phi_{1}=\Gamma t+\gamma
$$

where $\Gamma=K \frac{H-\left(s_{1}^{2} / c_{1}\right)}{K^{2}-s_{1}^{2}}$ and $\gamma$ is a constant of integration. Then substituting (50) and (48) into (39) yields:

$$
\begin{gathered}
a=\frac{r}{K}, \quad b=\frac{s_{1}}{K}, \quad c=\sin C t, \quad d=r \cos C t \\
e=\sin (\Gamma t+\gamma) \quad f=\cos (\Gamma t+\gamma)
\end{gathered}
$$

which defines the attitude motion at the singularity of the extremal curves completely analytically.

\section{CONCLUSION}

This paper has derived the analytic form of an optimal attitude motion at a singularity. This provides an analytic expression for an optimal motion in terms of the angular velocity history and the corresponding rotation matrix. This expression can then be used in a parametric optimization problem, where by the available parameters (the initial angular velocities) can be optimized to match boundary conditions such as initial and final pointing directions.

\section{REFERENCES}

Wie, B. (1998). Space Vehicle Dynamics and Control. AIAA Education Series

Spindler, K. (1996). Optimal Attitude Control of a Rigid Body Applied Mathematics and Optimization, 34: 79-90

Jurdjevic, V. (1997). Geometric Control Theory. Advanced Studies in Mathematics, Cambridge University Press, 52.

Leonard, N., Krishnaprasad, P. S. (1995). Motion control of drift free, left-invariant systems on Lie groups IEEE Transactions on Automatic control, vol. 40, pp.1539-1554

Sussmann, H. J., (1997). An introduction to the coordinatefree maximum principle In Geometry of Feedback and Optimal Control,B. Jakubczyk and W. Respondek Eds., Marcel Dekker, New York, pp. 463-557

Biggs, J. D., Holderbaum, W. (2010) Integrable quadratic Hamiltonians on the Euclidean group fo motions Journal of Dynamcial and Control systems, vol. 16, no. 3, pp. 301-307

Whittaker, E. T. (1999) A treatise on the analytical dynamics of particles and rigid bodies Cambridge Mathematical Library, Cambridge University Press 